\title{
THE IMPROVEMENT OF THE RHEOLOGICAL MODEL FOR CONTROLLING THE STRESS-STRAIN STATE AND HUMIDITY IN THE MATERIALS OF MUSEUM PIECES: ZENER THERMODIFFUSION MODEL FOR CAPILLARY- POROUS BODIES
}

\begin{abstract}
The drying and humidifying processes of capillary-porous (colloid) bodies occur during the production of various materials for the consumer goods industry, building materials, storage of museum pieces, etc. It is known that the main linkage forms of the moisture and colloid capillaryporous bodies (CCPBs), namely adsorption, capillary condensation and capillary linkage of the free moisture in the cavities of the above bodies, depend on the temperature and relative humidity of drying/humidifying agent. It means that the CCPBs behave in a peculiar way depending on the temperature and humidity fields. The problems of CCPBs drying (or humidifying) process include the issue of the heat and humidity transfer both in the middle of the body and in the boundary layer on the interface of phases "body (object of drying/humidifying process) - environment". The drying/humidifying intensity is at its maximum when the possibilities of the heat and mass transfer in the boundary layer correspond to the possibilities of moisture and heat moving inside the object of drying/humidifying process. The properties of the CCPB as material possessing specific elastic-viscous properties are described within the framework of O.Y. Ishlinskiy - O.R. Rzhanitsin generalized elasticviscous body theory. A comparative analysis of the above mentioned CCPB's properties from the point of view of Zener thermodiffusion theory is conducted. The mechanism of moisture transferring from the CCPBs' central layers (as object of drying/humidifying process) up to their surfaces is developed. The theoretical researches examined the drying/humidifying phenomena for both the entire volume of the CCPB and three structural directions. It is noted that drying/humidifying of CCPBs is a complicated heat and mass transfer process accompanied by mechanisms of molecular nature determining the kinetics of their running. It is shown that the appropriate equations' solution of molecular-molar heat and moisture transfer under the appropriate boundary (limit) conditions allows to describe the fields, i.e. the distribution of transfer potentials (the temperature and moisture content in the CCPB as object of drying/humidifying) at any time of the appropriate process. The drying/humidifying curves ("drying/humidifying rate versus CCPB humidity") and the temperature curves ("СCPB temperatures versus $\mathrm{CCPB}$ humidity") reflect the nature of the drying/humidifying processes. In the framework of the proposed generalized rheological model of CCPB, the residual deformations of bodies for various load types (the time-varying stress applied to the CCPB) is estimated.
\end{abstract}

Key words: aftereffect, colloid capillary-porous bodies, deformations, heat and mass transfer, Ishlinskiy Rzhanitsin generalized rheological model of the elastic-viscous body, Zener thermodiffusion rheological model.

\footnotetext{
${ }^{1}$ Candidate of Technical Sciences, professor, Kyiv National University of Construction and Architecture, Kyiv, Ukraine.

${ }^{2}$ Candidate of Technical Sciences, IPA profesor, National University of Life and Environmental Sciences of Ukraine, Kyiv, Ukraine, ychovnyuk@ukr.net.

${ }^{3}$ Senior Assistant, National Aviation University, Kyiv, Ukraine.

${ }^{4}$ Assistant, Kyiv National University of Construction and Architecture, Kyiv, Ukraine.
} 


\section{Introduction}

The colloid capillary-porous bodies (CCPBs) have elastic-viscous properties in the polymers' model limits, i.e. in the heated humid state, they are easily deformed as an elastic-viscous body, and in a dry state they react as an elastic body.

CCPBs' drying/humidifying process is a complex heat and mass transfer process accompanied simultaneously by external heat exchange (heat transfer from the drying/humidifying agent up to the surface CCPB's as object of drying/humidifying process), by internal heat exchange (heat transfer through CCPB due to heat conductivity) and humidity exchange (by evaporation/condensation of humidity from/on the surface of the CCPB) as well as by humidity transfer (displacement of humidity) inside the CCPB, i.e. from deep layers up to the surface of CCPB as object of drying/humidifying process.

The humidity coming from the surface zones of the CCPB evaporates into the environment faster than it moves from CCPBs' internal (central) layers to their surface (during drying processes) or in the inverse direction (during humidifying processes), then an unequal distribution of CCPB's humidity through the thickness of the material (CCPB) (object of drying/humidifying process) occurs.

In the opinion of authors of this research, the above drying/humidifying processes of $\mathrm{CCPBs}$ require their further comprehensive, in-depth study and analysis in order to create adequate physical-mechanical and mathematical models of such processes.

\section{Literature review}

The Ishlinskiy-Rzhanitsin generalized theory of the elastic-viscous body appeared due to the need to have a single description of the aftereffect and relaxation in solid bodies. Based on the same hypothesis, O.Y. Ishlinskiy and then O.R. Rzhanitsin: O.Y. Ishlinskiy built the theory of longitudinal oscillations of the elasticviscous rods [1], O.R. Rzhanitsin formulated the general theory of the elastic-viscous body after its hereditary properties as well as the stability and transverse oscillation of rods were researched [2].

According to this theory, the stress $\sigma$ and the deformation $\varepsilon$ are linked by the following dependence [3]:

$$
\sigma+\kappa \cdot \&=E \cdot \varepsilon+\kappa^{\prime} \cdot E \cdot \& \&=\frac{d \sigma}{d t}, \&=\frac{d \varepsilon}{d t},
$$


where:

$\kappa$ and $\kappa^{\prime}-$ constants of the material (time/period of stresses' relaxation and time/period after the deformation respectively).

When rates of and \& are low compared to $\sigma / \kappa$ and $\varepsilon / \kappa^{\prime}$, the Hooke's law with the long term [2] or relaxing [4] module $E$ is obtained from (1). Inversely, when of and \& are high relative to $\sigma / \kappa$ and $\varepsilon / \kappa^{\prime}$ respectively, we obtain the Hooke's law with instantaneous [2] or non-relaxing [4] module $H=\kappa^{\prime} / \kappa \cdot E$. In describing the aftereffect and relaxation, this theory also describes some internal friction [3].

Zener's thermodiffusion theory [5] attributes the internal friction in materials to the thermal diffusion.

In referring in their fundamental work [6] to Zener's theory for more complete description of energy dissipation in solid bodies, the friction of thermodiffusion nature was added by L.D. Landau and E.M. Lifshitz to Kelvin-Voigt [3] viscous friction.

The physical foundations of the internal friction theory caused by the thermodiffusion are as follows.

As is well known, when the deformation is due to the compression, the body's temperature rises and if the tensile deformation takes place the temperature decreases. The cyclic tension-compression strains unbalance the body's temperature. This results in irreversible heat losses equivalent to the internal friction effect. Thus, if a cylindrical body (model of a CCPB's fibre) is exposed to the cyclic transverse bending, if the compressed fibres are heated and the stretched fibres are cooled, then, due to the temperature gradient, it is possible that the heat flow passes across the body and periodically changes its direction. If the period of deformation cycles is long enough allowing the temperature to equalize at the cross-section at any time, it means that the heat transfer process is carried out isothermally and accordingly, vice versa, without heat inputs. If the period of the deformation cycles is so short that the temperature fails to react to deformations, then the heat transfer process is impossible at all and deformations occur in adiabatic conditions, and, accordingly, vice versa again, without heat inputs. If the period of deformation cycles is of the same order as the time of passage of the heat flow across the body (the deformations occur in intermediate conditions between isothermal and adiabatic), then irreversible heat losses are possible which is characteristic of the internal friction.

Based on this reflections, Zener discovers the following dependence between the stress $(\sigma)$ and deformation $(\varepsilon)$ :

$$
\sigma+\tau \cdot \mathbb{\&}=E_{T} \cdot \varepsilon+\tau \cdot E_{S} \cdot \&
$$


where:

$\tau$ - time/duration (period) of temperature relaxation,

$E_{T}$ and $E_{S}$ - isothermal and adiabatic elasticity modulus respectively.

In Ishlinskiy - Rzhanitsin theory of the elastic-viscous body, the equation (2) is quite analogous to the equation (1). The time/duration (period) of temperature relaxation $\tau$ corresponds to the time of stress relaxation $\kappa$, isothermal and adiabatic elasticity modulus $E_{T}$ and $E_{S}$ corresponds to long term and instantaneous elasticity modulus $E$ and $H$. These data indicate that Zener's theory does not provide qualitatively new results in comparison with the generalized theory of elastic-viscous body.

The theory of CCPB's drying/humidifying process is based on the heat and humidity transfer theory quite thoroughly developed by academician O.V. Lykov and his followers for the processes of heat and mass exchange [7]. Based on the provisions of the heat and mass transfer theory, an equation was obtained for the analysis of experimental data of (wet) materials' drying/humidifying process as convective only and convective-radiant as well. In particular, based on the Ishlinskiy-Rzhanitsin model, the research paper [8] proposes a procedure for controlling the stress-strain state and humidity of wood in heat and mass transfer of drying process.

The results of scientific publications cited above will be partially used in this research.

The research's goal is to demonstrate the physical-mechanical (rheological) and mathematical models of heat and mass transfer processes of CCPB's drying/humidifying in order to control their stress-strain state and temperature/humidity parameters.

\section{Main concepts}

The humidity from surface zones of CCPB evaporates faster into the environment compared with the movement of humidity from internal (central) layers of the object of drying to the surface (and, conversely, the humidity from surface zones of the CCPB is absorbed faster by the material during processes of condensation compared to the movement of humidity from surface layers of the humidified object to its internal layers), then the thickness of the CCPB (object of drying/humidifying process) causes an unequal distribution of the moisture content of the material, then an unequal distribution of material's humidity through the thickness of the CCPB (object of drying/humidifying process) takes place. 
The size and shape of CCPB's block and of sheet materials change with the temperature change of the drying/humidifying agent (heated air, water vapour, etc.) and with the humidity change of the object of drying/humidifying process.

Generally, CCPB's drying/humidifying process is varying through the thickness of the object of drying/humidifying process. Drying of CCPBs, as well as their humidifying subject to variable heat and mass transfer potential is accompanied by the presence of a residual stress-strain state (SSS), as well as by the change in most of the CCPB's physical-mechanical properties. Significant internal stresses are a major constraint for the intensification of the CCPB's drying and humidifying processes. The solution of this scientific issue is complicated by the fact that CCPBs belong to the class of physically nonlinear bodies characterized by their high hydrophilicity, by significant variability of structural and physical-mechanical properties.

Presently, fundamentally important results of domestic and foreign scientists' studies do not fully reflect a complicated and multifaceted situation of the deformation-relaxation processes' development in CCPBs, because they do not take into account the relation between heat and mass transfer characteristics and the real rheological behaviour of materials. In particular, researches on the process of convection and radiation-convective drying of $\mathrm{CCPBs}$ shows, that the tensile strength along the fibres of the material is approximately the same for all methods of its drying, and the study of the SSS of CCPBs during the process of drying/humidifying allows to establish the rheological behaviour of materials having creep, relaxation and inverse creep, taking into account anisotropy within the humidity changing range $W=(8 \ldots 60) \%$ and $t=(20 \ldots 35)^{\circ} \mathrm{C}$.

Subject to the single-axis SSS of isotropic CCPBs, whose properties are the same in all directions, the Hooke's law can be presented as follows:

$$
\varepsilon=\frac{\sigma}{E}
$$

where:

$\varepsilon$ - relative longitudinal (linear) deformation of CCPB;

$\sigma-$ normal stress;

$E$ - elasticity modulus (the value is inversely proportional to the volumetric compression coefficient of the material, i.e.: $E=1 / \beta_{v} ; \beta_{v}=1 / V \cdot \partial V / \partial P$,

$V$ - volume,

$P$ - pressure, stress in the material); $[\sigma]=[E]=\mathrm{Pa} ;\left[\beta_{v}\right]=\mathrm{Pa}^{-1} ;[V]=\mathrm{m}^{3}$. 
Subject to orthogonal scheme of CCPB's elastic anisotropy, the planes of elastic symmetry are two longitudinal (radial and tangential) relative to (circular) layers of material's fibres and one plane is perpendicular to the fibre direction. Normals to these planes coincide with the direction of the coordinate axis, which have the following notation: $a(\mathrm{x})$ - along CCPB's fibres, $\tau(\mathrm{y})$ - radial direction across CCPB's fibres, $\tau(\mathrm{z})$ - tangential direction across CCPB's fibres (Fig. 1).

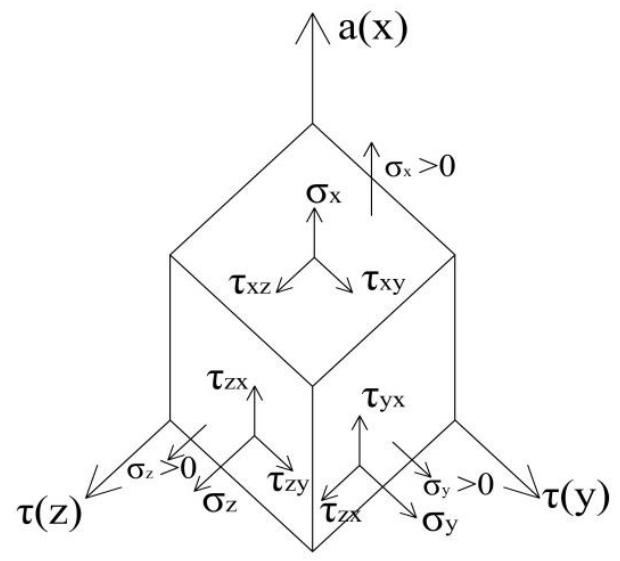

a)

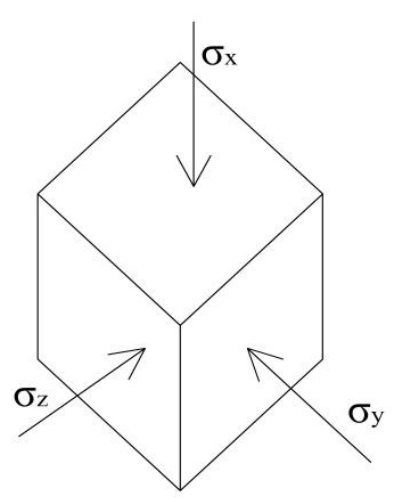

b)

Fig. 1. Main directions of the elastic symmetry of CCPB as orthotropic body: a) notation of stresses in the general case; $b$ ) stressed state of the triaxial equal compression.

Then, for anisotropic CCPBs, in which the value of the deformation depends not only on the value of the existing stresses, but also on the direction of their effect in the material, the Hooke's law will be expressed as follows:

$$
\begin{aligned}
& \varepsilon_{x}=\frac{\sigma_{x}}{E_{x}}-\frac{\mu_{y x} \cdot \sigma_{y}}{E_{y}}-\frac{\mu_{z x} \cdot \sigma_{z}}{E_{z}} ; \varepsilon_{y}=-\frac{\mu_{x y} \cdot \sigma_{x}}{E_{x}}+\frac{\sigma_{y}}{E_{y}}-\frac{\mu_{z y} \cdot \sigma_{z}}{E_{z}} ; \\
& \varepsilon_{z}=-\frac{\mu_{x z} \cdot \sigma_{x}}{E_{x}}-\frac{\mu_{y z} \cdot \sigma_{y}}{E_{y}}+\frac{\sigma_{z}}{E_{z}} ; \gamma_{x y}=\frac{\tau_{x y}}{G_{x y}} ; \gamma_{y z}=\frac{\tau_{y z}}{G_{y z}} ; \gamma_{z x}=\frac{\tau_{z x}}{G_{z x}},
\end{aligned}
$$

where:

$E_{x, y, z}-\mathrm{CCPB}$ 's elasticity modulus (along the corresponding axis $\mathrm{Ox}, \mathrm{Oy}, \mathrm{Oz}$ ); 
$G_{x y}, G_{y z}, G_{z x}-$ CCPB's shearing modulus of elasticity (along the corresponding plane $\mathrm{xOy}, \mathrm{yOz}, \mathrm{zOx})$;

$\mu$ - transverse deformation coefficient or Poisson's coefficient (the first index for $\mu$ indicates the direction of the transverse deformation and the second one indicates the direction of deformation causing its axial force);

$\gamma$ - angular stains; $\varepsilon$ - deformation along the axes (Ox, Oy, Oz).

Double indexes for the shearing modulus of elasticity $G_{i j},(i, j)=(\mathrm{x}, \mathrm{y}, \mathrm{z})$ correspond to the directions between which the change of the right angle occurs. Consequently, in order to establish relations between stress tensors and deformations, it is necessary to have 12 elastic constants between which 9 constants are independent.

For large-sized sheet materials (such as CCPB) in which it is impossible to neglect the curvature of layers, it is necessary to use the scheme of transversal isotropy, i.e. when in the plane perpendicular to the fibres, the elastic properties of CCPBs are similar in all directions. In other words, when the differences between properties along and across fibres are taken into account. Then for a transversal isotropic CCPB, the correlation between stress tensors and deformations is carried out using 5 elastic constants. Consequently, in order to establish SSS of CCPBs on the basis of the elasticity theory, it is necessary to know the numerical values of the material's elastic constants.

The main carrier of CCPB's mechanical properties is its anisotropic component. If the material is not loaded along the fibres, two zones of deformation can be distinguished, one of which is the zone of normal elasticity and the second one is the zone of forced elastic deformation (the latter consists mainly of thermoreversible residual deformations). Then the stresses that determine the boundary between the abovementioned zones will be the boundary of the CCPB's forced elasticity. In other words, if the tension in the second zone affects the swollen CCPB, then it will come out of the state of natural "glazing" and will acquire highly elastic properties. It is worth noting, that high elastic deformations are inverse and dozens of times exceed instantaneous elastic deformations of the CCPB.

Consequently, a rheological model can be presented as model consisting of successively connected two models: a Hooke elastic body (symbolically represented by an elastic model with the elasticity modulus $E_{2}$ ), of the Kelvin elastic body (symbolically represented in the form of springs connected in parallel (elasticity modulus $E_{1}$ ) and of the damper with a fluid having a viscosity $\eta_{v}$ ), [ $\left.\eta_{v}\right]=\mathrm{Pa} \cdot \mathrm{s}$ (Fig. 2). 


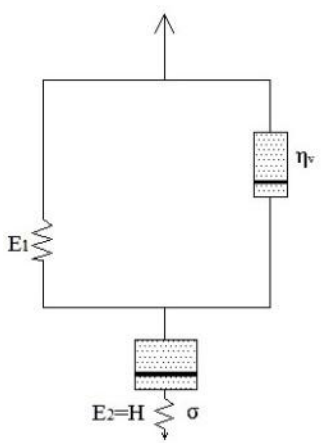

a)

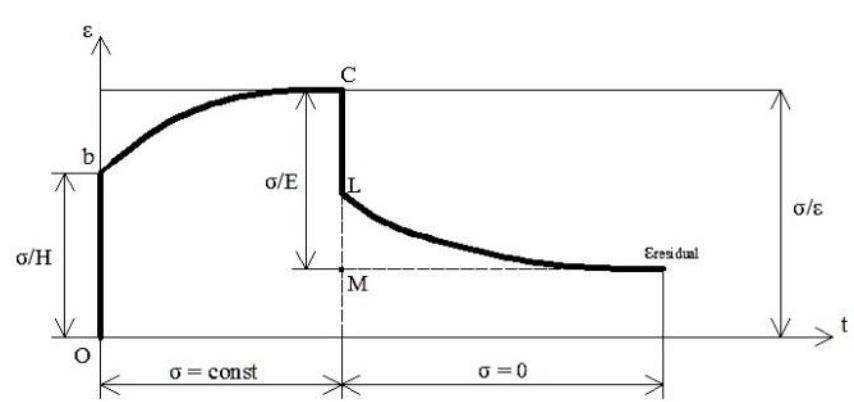

b)

Fig. 2. Rheological model of CCPB: a) scheme; b) regularities of its deformation.

For such a model, the dependence of the stress $\kappa$ and deformation $\varepsilon$ will be described by the equation:

$$
\sigma+n \cdot \&=n \cdot H \cdot \&+E \cdot \varepsilon,
$$

where:

$H=E_{2}-$ instantaneous elasticity modulus;

$E$ - long-term elasticity modulus $\left(E=\left(E_{1} \cdot E_{2}\right) /\left(E_{1}+E_{2}\right)\right)$;

$n$-relaxation duration $\left(n=\eta_{8} /\left(E_{1}+E_{2}\right)\right),[n]=\mathrm{s}$.

In the case of application of the load a deformation appears instantaneously $\varepsilon=\frac{\sigma}{H}$. Next, under constant stress $(\sigma=$ const $)$, elastic deformations increase according to the curvilinear law (see Fig. 2,b), and due to the long exposure, the deformation tends to a value equal to $\varepsilon=\sigma / E$. After unloading, an elastic deformation returns immediately and then resilient deformation disappears. Consequently, this model fully reflects the behaviour of the $\mathrm{CCPB}$, the deformation of which is completely reversible.

Under the conditions of short-term effect of relatively insignificant loads, a CCPB behaves like an elastic body. The deformation determines the change in the linear and angular dimensions of the body and characterizes the state of its particular segments. The temporal and residual deformations and stresses are determined in a simplest way for the case of single-axis stress state observed during the heat supply to long narrow strips or during the thermal treatment of elements' rectilinear edges. 
The deformations in the $\mathrm{CCPB}$ are manifested in the direction transverse to the fibre length (as a matter of fact, it allows to apply Ziner's model or similar in substance Ishlinskiy-Rzhanitsin model). There can be elastic deformations of CCPB that, during long term drying/humidifying process (heat/humidity supplying), causes the deformation of elastic aftereffect (sometimes they are called elastic-resilient) and residual (irreversible) deformations with their simultaneous running in time in the cross-section of the drying/humidifying agent. In such event, under the effect of elastic deformations, a CCPB (being an elastic-viscous body) can be residually stretched or compressed, especially in a wet and heated state. In this case, the elastic deformation becomes spontaneously the residual one fixing the new size of the object of drying/humidifying process (it is characteristic for the first stage of drying/humidifying process). At the second stage of drying/humidifying process, phenomena inverse to those described above occur, i.e. the residual tensile deformation appears in the internal zone of CCPB's layers and in the external zone, the compressive deformation takes place (during drying process) and it can occur vice versa if the humidifying process is described.

For example, a gradual decrease (relaxation) of tensile elastic deformations in CCPB's central layers (during drying process) and of compressive deformations in surface (external) layers occur also after final drying. In other words, the essence of relaxation is precisely the transition of elastic deformations (i.e. stresses in the CCPB) in residual deformations. In addition, the relaxation itself follows first the steeply dipping (exponential) curve, and then approaches its horizontal asymptotes (see Fig. 2,b). For a unit of relaxation time a period during which the value of initial stress decreases in $e=2,72$ times is taken. This time is determined by the formula:

$$
\tau^{*}=\frac{\eta_{v}}{E_{1}+H}=\frac{\eta_{v}}{E_{1}+E_{2}}=A \cdot \exp \left\{-\frac{U_{a}}{k \cdot T}\right\}
$$

where:

$\eta_{v}$ - CCPB's viscosity coefficient;

$H$ - instantaneous elasticity modulus in the direction of loading;

- additional elastic linkage;

$k$ - Boltzmann constant;

$U_{a}$ - activation energy of the relaxation process;

$T$ - body's absolute temperature;

A - coefficient. 
In the case of constant loading $\sigma$ at the time point $t$, the development of deformation $\varepsilon$ is given in Fig. 2,b (graph at point C). At point $\mathrm{C}$, the load is instantaneously thrown off, then the segment CL will show an elastic deformation and the segment LM demonstrates a deformation of elastic aftereffect, i.e. resilient deformation. Then taking into account these considerations, this equation will be valid:

$$
\varepsilon \cdot\left(\tau^{*} \cdot H+E\right)=\sigma_{t=\tau^{*}+1},
$$

where:

$\varepsilon$ - relative deformation;

$E$ - elasticity modulus in the direction of loading.

In case, when $\tau \approx 0, \sigma \approx \varepsilon \cdot E$ (mostly elastic deformation occurs).

The residual deformation $\varepsilon_{\text {residual }}$ is determined by the equation:

$$
\varepsilon_{\text {residual }}=\frac{\left.\sigma\right|_{t=\tau^{*}+1}}{\tau^{*} \cdot H+E}=\frac{\left.\sigma\right|_{t=\tau^{*}+1}}{\tau^{*} \cdot E_{2}+E} .
$$

According to Ishlinskiy-Rzhanitsin model (1) and Zener model (2), the coefficients of the model (5) are determined as follows:

$$
\tau^{*}=\frac{\eta_{6}}{E_{1}+E_{2}}=n=\tau ; H=E_{2}=E_{S} ; E=\frac{E_{1} \cdot E_{2}}{E_{1}+E_{2}}=E_{T},
$$

where:

$\tau$ - time (period) of temperature relaxation (according to Zener),

$E_{S}-$ CCPB's adiabatic elasticity modulus,

$E_{T}$ - CCPB's isothermal modulus of elasticity.

Consequently, Zener model (1) can be further used.

Let's consider the analogous dependences $\varepsilon(t)$ if the dependence $\sigma(t)$ has the following form (Fig. 3). 


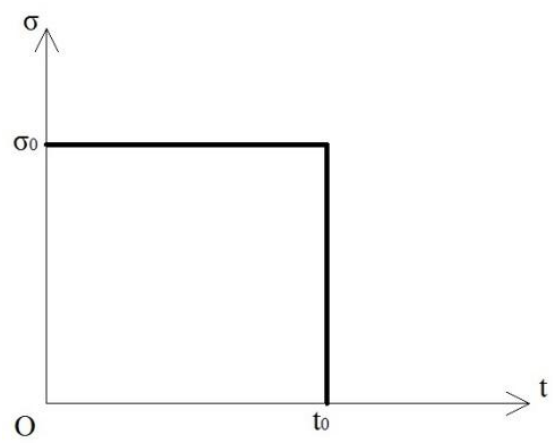

Fig. 3. Dependence $\sigma(t)$

The first stage. In case of load application $\sigma_{0}=$ const during the time $t \in\left[0 ; t_{0}\right]$ an elastic deformation only appears first:

$$
\varepsilon_{0}=\frac{\sigma_{0}}{H}=\frac{\sigma_{0}}{E_{S}} .
$$

The second stage. Subsequently, for $\sigma_{0}=$ const, the resilient deformations increase according to the curvilinear law:

$$
\sigma_{0}=E_{T} \cdot \varepsilon+\tau \cdot E_{S} \cdot \& \Leftrightarrow \& \frac{E_{T}}{E_{S} \cdot \tau} \cdot \varepsilon=\frac{\sigma_{0}}{E_{S} \cdot \tau},
$$

That is the solution of the equation (11), i.e.:

$$
\varepsilon\left(t_{0}\right)=\sigma_{0}\left(\frac{1}{E_{S}}-\frac{1}{E_{T}}\right) \cdot \exp \left\{-\frac{E_{T}}{E_{S} \cdot \tau} \cdot t\right\}+\frac{\sigma_{0}}{E_{T}} .
$$

Due to the long term exposure of the CCPB, its deformation tends to the value equal to: $\varepsilon_{\infty}=\sigma_{0} / E_{T}$.

The third stage. After instantaneous unloading of the CCPB immediately (at the time point $t=t_{0}$ ) elastic deformation returns:

$$
\begin{aligned}
\varepsilon\left(t_{0}\right) & =\sigma_{0}\left(\frac{1}{E_{S}}-\frac{1}{E_{T}}\right) \cdot \exp \left\{-\frac{E_{T}}{E_{S} \cdot \tau} \cdot t_{0}\right\}+\frac{\sigma_{0}}{E_{T}}-\frac{\sigma_{0}}{E_{S}}= \\
& =\sigma_{0}\left(\frac{1}{E_{T}}-\frac{1}{E_{S}}\right) \cdot\left\{1-\exp \left[-\frac{E_{T}}{E_{S} \cdot \tau} \cdot t_{0}\right]\right\} .
\end{aligned}
$$


Considering, that $E=\left(E_{1} \cdot E_{2}\right) /\left(E_{1}+E_{2}\right), E<E_{2}$, it means that $E_{T}<E_{\mathrm{S}}$.

The fourth stage. In the future, the aftereffects process is put into action for $\varepsilon(t)$, $t>t_{0}$, considering that it cannot instantly fall to zero due to the inertance of all physical processes. At this stage, the deformation is determined by the following equation:

$$
\tau \cdot E_{S} \cdot \&+E_{T} \cdot \varepsilon=0,\left.\varepsilon\right|_{t_{0}}=\varepsilon\left(t_{0}\right)=\sigma_{0}\left(\frac{1}{E_{T}}-\frac{1}{E_{S}}\right) \cdot\left\{1-\exp \left[-\frac{E_{T}}{E_{S} \cdot \tau} \cdot t_{0}\right]\right\} .
$$

Then:

$$
\left\{\begin{array}{l}
\varepsilon(t)=\sigma_{0}\left(\frac{1}{E_{T}}-\frac{1}{E_{S}}\right) \cdot\left\{1-\exp \left[-\frac{E_{T}}{E_{S} \cdot \tau} \cdot t_{0}\right]\right\} \cdot \exp \left\{-\frac{E_{T}}{E_{S} \cdot \tau} \cdot t\right\}, \\
t>t_{0} .
\end{array}\right.
$$

The value of residual deformation will be determined for the following reasons. The new variable $t^{\prime}=t-t_{0}$ is put in. It is necessary to determine the period of time $\left(t^{\prime}\right)^{*}=\Delta$ during which the value $\varepsilon(t)(15)$ will decrease by a factor of 10 , i.e.:

$$
\left.\varepsilon(t)\right|_{t=t_{0}+\Delta}=\left.\left(\frac{1}{10}\right) \cdot \varepsilon(t)\right|_{t=t_{0}},
$$

or:

$$
\left.\varepsilon\left(t^{\prime}\right)\right|_{t^{\prime}=\Delta}=\left.\frac{1}{10} \cdot \varepsilon\left(t^{\prime}\right)\right|_{t^{\prime}=0} .
$$

Then, for $\Delta$ the following equation:

$$
\exp \left\{-\frac{E_{T}}{E_{S} \cdot \tau} \cdot \Delta\right\}=\frac{1}{10}
$$

It results from (18):

$$
\Delta=\frac{E_{S} \cdot \tau}{E_{T}} \cdot \ln 10
$$


Thus, for the residual deformation $\varepsilon_{\text {residual, }}$ the law $\kappa(t)$ (Fig. 3) is as follows:

$$
\left.\varepsilon_{\text {residual }}\right|_{t=t_{0}+\Delta}=\sigma_{0}\left(\frac{1}{E_{T}}-\frac{1}{E_{S}}\right) \cdot\left\{1-\exp \left[-\frac{E_{T}}{E_{S} \cdot \tau} \cdot t_{0}\right]\right\} \cdot \exp \left\{-\frac{E_{T}}{E_{S} \cdot \tau} \cdot\left(t_{0}+\Delta\right)\right\} \text {. }
$$

In this case, $\Delta$ in (20) is determined by the equation (19). Since $\tau$ is the duration (time)/ period of temperature relaxation (according to Zener), the equation (20) can be alternatively presented as follows:

$$
\left.\varepsilon_{\text {residual }}\right|_{t=t_{0}+\Delta}=\sigma_{0}\left(\frac{1}{E_{T}}-\frac{1}{E_{S}}\right) \cdot\left\{1-\exp \left[-\frac{E_{T}}{E_{S} \cdot \tau} \cdot t_{0}\right]\right\} \cdot \exp \left\{-\frac{E_{T} \cdot t_{0}}{E_{S} \cdot \tau}\right\} \cdot \frac{1}{10} \text {. }
$$

The dependence $\varepsilon(t)$ will be further examined if $\sigma(t)$ varies according to the law given in the Fig. 4.

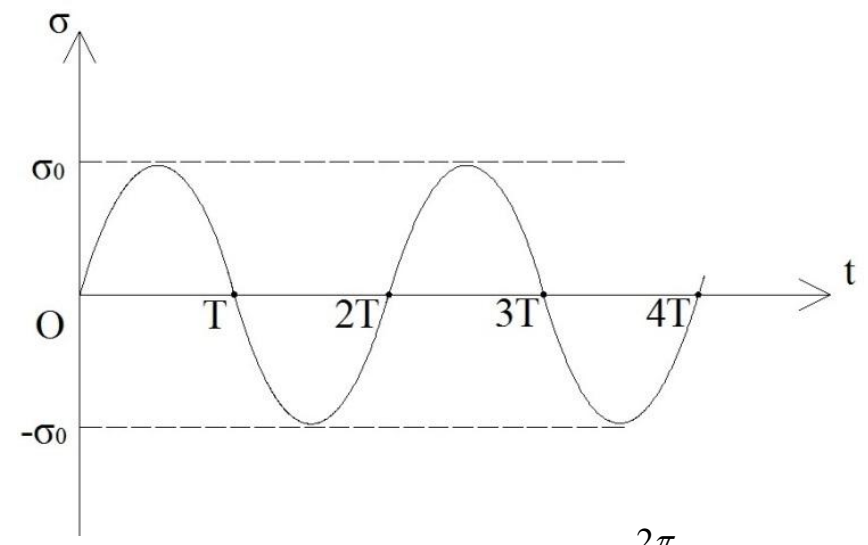

Fig. 4. Dependence $\sigma(t), T=\frac{2 \pi}{\omega}$.

Supposing, that Zener rheological model is valid for this law $\sigma(\mathrm{t})$ (Fig. 4). In this case: $(\varepsilon, \sigma) \sim \exp (i \omega \tau), i^{2}=-1$. Then, Zener rheological model (2) becomes as follows:

$$
\sigma \cdot(1+i \cdot \omega \cdot \tau)=\varepsilon \cdot\left(E_{T}+i \cdot \omega \cdot \tau \cdot E_{S}\right)
$$


Then:

$$
\begin{gathered}
\varepsilon(t)=\frac{\sigma(t) \cdot(1+i \cdot \omega \cdot \tau) \cdot\left(E_{T}-i \cdot \omega \cdot \tau \cdot E_{S}\right)}{\left(E_{T}^{2}+\omega^{2} \cdot \tau^{2} \cdot E_{S}^{2}\right)} . \\
|\varepsilon(t)|=\frac{|\sigma(t)|}{\left(E_{T}^{2}+\omega^{2} \cdot \tau^{2} \cdot E_{S}^{2}\right)} \cdot\left\{\left(E_{T}+\omega^{2} \cdot \tau^{2} \cdot E_{S}\right)^{2} \cdot\left(E_{S}-E_{T}\right)^{2} \cdot \omega^{2} \cdot \tau^{2}\right\}^{1 / 2} .
\end{gathered}
$$

In this case, $\varepsilon(t)$ is displaced in phase relative to $\kappa(t)$ by the angle $\varphi$, which is determined from the equation:

$$
\varphi=\operatorname{arctg}\left\{\frac{\omega \cdot \tau \cdot\left(E_{T}-E_{S}\right)}{\left(E_{T}+\omega^{2} \cdot \tau^{2} \cdot E_{S}\right)}\right\} .
$$

Considering that $E_{T}<E_{S}$, then $\varphi<0$, i.e. there is a time delay $t$ of the deformation $\varepsilon(t)$ relative to the law of change in time $\sigma(t)$.

Taking into account (24) and (25), $\varepsilon(t)$ is finally presented as follows:

$$
\varepsilon(t)=|\varepsilon(t)| \cdot \exp \{i \cdot \varphi\}
$$

The valid component $\varepsilon(t)$ is as follows:

$$
\operatorname{Re}\{\varepsilon(t)\}=|\varepsilon(t)| \cdot \cos \varphi
$$

There are several situations to consider.

Situation 1. $\omega \cdot \tau$ ? 1, i.e. $\omega$ ? $1 / \tau$ and the frequency of change in time $\sigma(t)$ is much higher than the inverse value of the temperature relaxation period $\tau$.

In this case: $\varphi \rightarrow(-0)$, and $\varepsilon(t) \rightarrow \sigma(t) / E_{S}$.

Situation 2. $\omega \cdot \tau=1$, i.e. $\omega=1 / \tau$. In this case: $\varphi \rightarrow(-0)$, a $\varepsilon(t) \rightarrow \sigma(t) / E_{S}$.

Situation $3 . \omega \cdot \tau: 1$, then:

$$
\left\{\begin{array}{l}
\varphi=\operatorname{arctg}\left\{-\frac{\omega \cdot \tau \cdot\left(-E_{T}+E_{S}\right)}{\left(E_{T}+\omega^{2} \cdot \tau^{2} \cdot E_{S}\right)}\right\}=\operatorname{arctg}\left\{-\frac{\omega \cdot \tau \cdot\left(E_{S}-E_{T}\right)}{E_{T}+\omega^{2} \cdot \tau^{2} \cdot E_{S}}\right\} \\
\varepsilon(t)=\frac{\sigma(t) \cdot \cos \varphi \cdot\left(1+\omega^{2} \cdot \tau^{2}\right)^{1 / 2}}{\left[E_{T}^{2}+\omega^{2} \cdot \tau^{2} \cdot E_{S}^{2}\right]^{1 / 2}}=\sigma(t) \cdot \cos \varphi \cdot \sqrt{\frac{\left(1+\omega^{2} \cdot \tau^{2}\right)}{E_{T}^{2}+\omega^{2} \cdot \tau^{2} \cdot E_{S}^{2}}} .
\end{array}\right.
$$


Considering, that the following case when $\sigma(t)$ varies according to the law given in Fig. 5, i.e.:

$$
\sigma(t)=\sigma_{0} \cdot \exp (-\lambda t)
$$

where $\lambda^{-1}$ - duration (time)/period of stress relaxation.

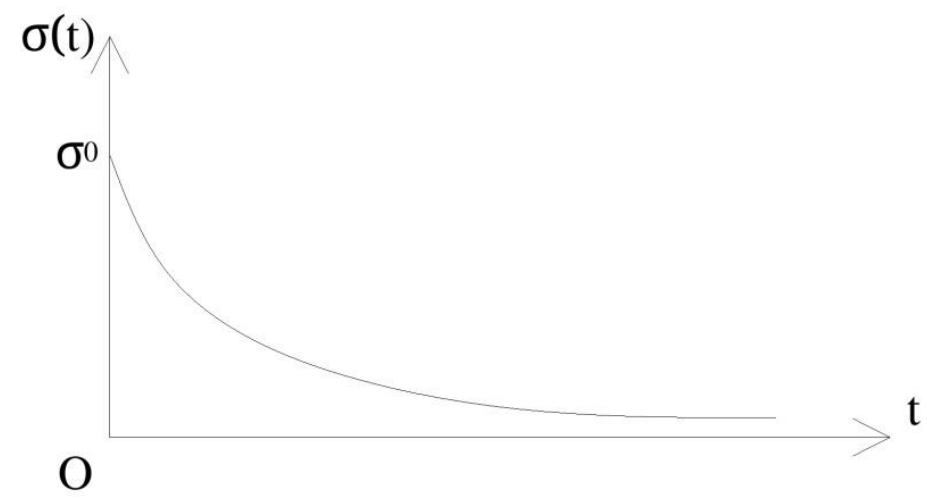

Fig. 5. The law of change in time $\sigma(t)$ (for the stress relaxation).

Then, in accordance to Zener rheological model:

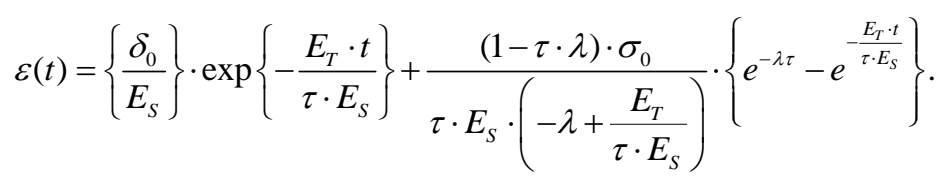

If $\lambda=1 / \tau$, then with (30):

$$
\varepsilon(t)=\left\{\frac{\sigma_{0}}{E_{S}}\right\} \cdot \exp \left\{-\frac{E_{T} \cdot t}{\tau \cdot E_{S}}\right\} .
$$

The effect of the humidity gradient in the CCPB during the drying/humidifying process is further considered. The presence of such a gradient generates also residual deformations in the material.

The sorption characteristic of the CCPB (i.e. sorption-desorption isotherm) which describes the dependence of moisture rate $(W, \%)$ absorbed by the material on relative humidity $(\varphi, \%)$ of rooms/environment where the CCPB is located. This can be conditionally presented by a hysteresis curve divided into three segments: 
segment I characterizes monomolecular adsorption, segment II characterizes polymolecular adsorption, segment III characterizes capillary and osmotically retained moisture in the CCPB.

Type I dependence $W(\varphi)$ conditionally corresponds to values $\varphi=(0 \ldots 10) \%$, type II corresponds to $\varphi=(10 \ldots 70) \%$, type III corresponds to $\varphi=(70 \ldots 100) \%$. Such a methodology for dividing sorption-desorption isotherms into segments (I, II, III) having a different mechanism of filling CCPB's pores with moisture, is used in research papers [9-12]. This approach allows to analyse the differential humidity transfer properties of CCPB in the hygroscopic area. To do this, the graph [13, Fig. 4.6] is used. The dependences $W(\varphi)$ and $\varepsilon(\varphi)$ (where $\varepsilon$-deformation of the CCPB) obtained experimentally for each segment I, II, and III of those areas are presented there.

The above dependences $W(\varphi)$ and $\varepsilon(\varphi)$ are given for each section (I, II, III) in the form of splines of the third order with respect to $\varphi$ (separately for the sorption stage (s) and for the desorption stage (d)). Then, these dependences obtain the following form:

Segment I:

$$
\left\{\begin{array}{l}
W_{I s}(\varphi)=a_{0 s}^{(1)}+a_{1 s}^{(1)} \cdot \varphi^{1}+a_{2 s}^{(1)} \cdot \varphi^{2}+a_{3 s}^{(1)} \cdot \varphi^{3} ; \\
W_{I d}(\varphi)=a_{0 d}^{(1)}+a_{1 d}^{(1)} \cdot \varphi^{1}+a_{2 d}^{(1)} \cdot \varphi^{2}+a_{3 d}^{(1)} \cdot \varphi^{3} \\
\varepsilon_{I s}(\varphi)=8_{0 s}^{(1)}+8_{1 s}^{(1)} \cdot \varphi^{1}+8_{2 s}^{(1)} \cdot \varphi^{2}+8_{3 s}^{(1)} \cdot \varphi^{3} \\
\varepsilon_{I d}(\varphi)=8_{0 d}^{(1)}+8_{1 d}^{(1)} \cdot \varphi^{1}+8_{2 d}^{(1)} \cdot \varphi^{2}+8_{3 d}^{(1)} \cdot \varphi^{3} .
\end{array}\right.
$$

Segment II:

$$
\left\{\begin{array}{l}
W_{I I s}(\varphi)=a_{0 s}^{(2)}+a_{1 s}^{(2)} \cdot \varphi^{1}+a_{2 s}^{(2)} \cdot \varphi^{2}+a_{3 s}^{(2)} \cdot \varphi^{3} ; \\
W_{I I d}(\varphi)=a_{0 d}^{(2)}+a_{1 d}^{(2)} \cdot \varphi^{1}+a_{2 d}^{(2)} \cdot \varphi^{2}+a_{3 d}^{(2)} \cdot \varphi^{3} ; \\
\varepsilon_{I I s}(\varphi)=8_{0 s}^{(2)}+8_{1 s}^{(2)} \cdot \varphi^{1}+8_{2 s}^{(2)} \cdot \varphi^{2}+8_{3 s}^{(2)} \cdot \varphi^{3} \\
\varepsilon_{I I d}(\varphi)=8_{0 d}^{(2)}+8_{1 d}^{(2)} \cdot \varphi^{1}+8_{2 d}^{(2)} \cdot \varphi^{2}+8_{3 d}^{(2)} \cdot \varphi^{3} .
\end{array}\right.
$$

Segment III:

$$
\left\{\begin{array}{l}
W_{I I I}(\varphi)=a_{0 s}^{(3)}+a_{1 s}^{(3)} \cdot \varphi^{1}+a_{2 s}^{(3)} \cdot \varphi^{2}+a_{3 s}^{(3)} \cdot \varphi^{3} ; \\
W_{I I I d}(\varphi)=a_{0 d}^{(3)}+a_{1 d}^{(3)} \cdot \varphi^{1}+a_{2 d}^{(3)} \cdot \varphi^{2}+a_{3 d}^{(3)} \cdot \varphi^{3} \\
\varepsilon_{I I I s}(\varphi)=8_{0 s}^{(3)}+8_{1 s}^{(3)} \cdot \varphi^{1}+8_{2 s}^{(3)} \cdot \varphi^{2}+8_{3 s}^{(3)} \cdot \varphi^{3} ; \\
\varepsilon_{I I I d}(\varphi)=8_{0 d}^{(3)}+8_{1 d}^{(3)} \cdot \varphi^{1}+\beta_{2 d}^{(3)} \cdot \varphi^{2}+8_{3 d}^{(3)} \cdot \varphi^{3} .
\end{array}\right.
$$


The coefficients of the equations (32) - (34) are determined taking into account conditions (we take $\varphi$ in relative unit fractions):

Segment I:

$$
\begin{aligned}
& \left.W_{I s}(\varphi)\right|_{\varphi=0}=0 ;\left.W_{I s}(\varphi)\right|_{\varphi=0,1}=W_{I s}(0,1) ; \\
& \left.\frac{d W_{I s}}{d \varphi}\right|_{\varphi=0}=\left.\left[W_{I s}(\varphi)\right]^{\prime}\right|_{\varphi=0} ;\left.\frac{d W_{I s}}{d \varphi}\right|_{\varphi=0,1}=\left.\left[W_{I s}(\varphi)\right]^{\prime}\right|_{\varphi=0,1} .
\end{aligned}
$$

Similar 4 conditions can be noted to determine spline coefficients in respect to $\varphi$ for $W_{I d}(\varphi)$ by replacing the index "s" with the index "d" in formulae (35). The right side of each equation (35) are completely determined by experimental values. The derivatives $W_{I s}(\varphi)$ and $W_{I d}(\varphi)$ with respect to $\varphi$ are elementarily determined:

$$
\left\{\begin{array}{l}
\frac{d W_{I s}}{d \varphi}=a_{1 s}^{(1)}+2 \cdot a_{2 s}^{(1)} \cdot \varphi+3 \cdot a_{3 s}^{(1)} \cdot \varphi^{2} ; \\
\frac{d W_{I d}}{d \varphi}=a_{1 d}^{(1)}+2 \cdot a_{2 d}^{(1)} \cdot \varphi+3 \cdot a_{3 d}^{(1)} \cdot \varphi^{2} .
\end{array}\right.
$$

Taking from the conditions (35) 4 linear equations for the coefficients $a_{0 s}^{(1)}, a_{1 s}^{(1)}, a_{2 s}^{(1)}, a_{3 s}^{(1)}$ (and in a similar way, for the coefficients

$\left.a_{0 d}^{(1)}, a_{1 d}^{(1)}, a_{2 d}^{(1)}, a_{3 d}^{(1)}\right)$

these coefficients are determined according to the Cramer's rule. $W_{I s}(\varphi)$ and $W_{I d}(\varphi)$ are completely defined in the form of spline approximation (cubic spline with respect to $\varphi$ ).

For the coefficients of functions $\varepsilon_{I s}(\varphi)$ and $\varepsilon_{I d}(\varphi)$, the procedure for determining the coefficients $\beta_{0 s}^{(1)}, \boldsymbol{\theta}_{1 s}^{(1)}, \boldsymbol{\theta}_{2 s}^{(1)}, \boldsymbol{\theta}_{3 s}^{(1)}$ (and accordingly $\boldsymbol{\theta}_{0 d}^{(1)}, \boldsymbol{\theta}_{1 d}^{(1)}, \boldsymbol{\theta}_{2 d}^{(1)}, \boldsymbol{\theta}_{3 d}^{(1)}$ ) is similar to the above procedure for the functions $W_{I s}(\varphi)$ and $W_{I d}(\varphi)$ with the difference that it is necessary to replace $W \rightarrow \varepsilon$ in (35) (and for finding coefficients, it is necessary to replace the index "s" with the index "d" in (35)) in equations for $\varepsilon_{I s}(\varphi)$.

For the functions $W_{I I s}(\varphi), W_{I I d}(\varphi), \varepsilon_{I I s}(\varphi), \varepsilon_{I d}(\varphi)$, coefficients are determined by equations: 


$$
\begin{aligned}
& \left.W_{I I s}(\varphi)\right|_{\varphi=0,1}=W_{I I s}(0,1) ;\left.W_{I I s}(\varphi)\right|_{\varphi=0,7}=W_{I I s}(0,7) ; \\
& \left.\frac{d W_{I I s}}{d \varphi}\right|_{\varphi=0,1}=\left.\left[W_{I I s}(\varphi)\right]^{\prime}\right|_{\varphi=0,1} ;\left.\frac{d W_{I I s}}{d \varphi}\right|_{\varphi=0,7}=\left.\left[W_{I I s}(\varphi)\right]^{\prime}\right|_{\varphi=0,7} ; \\
& \left.W_{I I d}(\varphi)\right|_{\varphi=0,1}=W_{I I d}(0,1) ;\left.W_{I I d}(\varphi)\right|_{\varphi=0,7}=W_{I I d}(0,7) ; \\
& \left.\frac{d W_{I I d}}{d \varphi}\right|_{\varphi=0,1}=\left.\left[W_{I I d}(\varphi)\right]^{\prime}\right|_{\varphi=0,1} ;\left.\frac{d W_{I I d}}{d \varphi}\right|_{\varphi=0,7}=\left.\left[W_{I I d}(\varphi)\right]^{\prime}\right|_{\varphi=0,7} .
\end{aligned}
$$

For the function $\varepsilon_{I I s}(\varphi), \varepsilon_{I I d}(\varphi)$ in the second segment in the equations of type (37), it is necessary to replace only $W \rightarrow \varepsilon$.

For the functions $W_{I I I s}(\varphi), W_{I I d}(\varphi), \varepsilon_{I I I s}(\varphi), \varepsilon_{I I I d}(\varphi)$, the coefficients are determined by the equations:

$$
\begin{aligned}
& \left.W_{I I I s}(\varphi)\right|_{\varphi=0,7}=W_{I I I s}(0,7) ;\left.W_{I I I s}(\varphi)\right|_{\varphi=1,0}=W_{I I I s}(1,0) ; \\
& \left.\frac{d W_{I I I s}}{d \varphi}\right|_{\varphi=0,7}=\left.\left[W_{I I I s}(\varphi)\right]^{\prime}\right|_{\varphi=0,7} ;\left.\frac{d W_{I I I s}}{d \varphi}\right|_{\varphi=1,0}=\left.\left[W_{I I I s}(\varphi)\right]^{\prime}\right|_{\varphi=1,0} ; \\
& \left.W_{I I I d}(\varphi)\right|_{\varphi=0,7}=W_{I I I d}(0,7) ;\left.W_{I I I d}(\varphi)\right|_{\varphi=1,0}=W_{I I I d}(1,0) ; \\
& \left.\frac{d W_{I I I d}}{d \varphi}\right|_{\varphi=0,7}=\left.\left[W_{I I I d}(\varphi)\right]^{\prime}\right|_{\varphi=0,7} ;\left.\frac{d W_{I I I d}}{d \varphi}\right|_{\varphi=1,0}=\left.\left[W_{I I I d}(\varphi)\right]^{\prime}\right|_{\varphi=1,0} .
\end{aligned}
$$

For the functions $\varepsilon_{I I I s}(\varphi), \varepsilon_{I I d}(\varphi)$, it is necessary to replace only $W \rightarrow \varepsilon$ in the segment III in the equations similar to (38).

The right side of equations (35), (37), (38) and analogous to them for functions $\varepsilon_{I s}(\varphi), \varepsilon_{I d}(\varphi), \varepsilon_{I I s}(\varphi), \varepsilon_{I I d}(\varphi), \varepsilon_{I I I s}(\varphi), \varepsilon_{I I d}(\varphi)$ are experimental (real) values obtained by the empirical way for certain temperatures' values and CCPBs' types.

The residual deformation occurring in the CCPB at a certain temperature due to changes in humidity parameters of the environment is determined for each segment (I, II, III) as follows:

$$
\left\{\begin{array}{l}
\varepsilon_{\text {residualI }}(\varphi)=\varepsilon_{I d}(\varphi)-\varepsilon_{I s}(\varphi) \\
\varepsilon_{\text {residualII }}(\varphi)=\varepsilon_{I I d}(\varphi)-\varepsilon_{I I s}(\varphi) \\
\varepsilon_{\text {residualIII }}(\varphi)=\varepsilon_{I I I d}(\varphi)-\varepsilon_{I I I s}(\varphi) .
\end{array}\right.
$$

If the temperature and moisture content $(W)$ of the CCPB changes simultaneously in connection with the flow of visitors in museum rooms, the total residual deformation of the CCPB's material has 2 components: a) the residual deformation of the CCPB due to the change of ambient temperature (formulae (20), 
(21), (27), (28), (30), (31)); b) the residual deformation of the CCPB due to the change of ambient air humidity.

Let the total residual deformation of the CCPB be at the initial time point $(t=0)$ (without visitors in museum rooms):

$\varepsilon_{\text {residual }}^{0}\left(\varphi_{0}, T_{0}\right)=\varepsilon_{\text {Id }}(\varphi)-\varepsilon_{\text {Is }}(\varphi)$, where $\varphi_{0}, T_{0}$ - relative humidity and absolute temperature $(\mathrm{K})$ of the air at the time point $t=0$.

At the time point $t=t^{*}>0$, in the presence of visitors in museum rooms, the temperature increased by $\Delta T$ and was equal to $T_{0}+\Delta T$, and the relative humidity in this room increased by $\Delta \varphi$ and was $\varphi_{0}+\Delta \varphi$. Then, total residual deformation of the $\mathrm{CCPB}$ at the time point $t^{*}$ is $\varepsilon_{\text {residual }}^{t^{*}}\left(\varphi_{0}+\Delta \varphi, T_{0}+\Delta T\right)$. The humidity increase is $\Delta \varphi=\varphi_{0}$ and the temperature increase is $\Delta T=T_{0}$. Then, the residual deformation of the CCPB at the time point $t=t^{*>0} 0$ has the following value (in a first approximation):

$$
\varepsilon_{r e s i d u a l}^{\left(*^{*}\right)}\left(\varphi_{0}+\Delta \varphi ; T_{0}+\Delta T\right)=\varepsilon_{\text {residual }}^{0}\left(\varphi_{0}, T_{0}\right)+\left.\frac{\partial \varepsilon_{r}}{\partial \varphi}\right|_{\varphi=\varphi_{0}: T=T_{0}} \cdot \Delta \varphi+\left.\frac{\partial \varepsilon_{r}}{\partial T}\right|_{\varphi=\varphi_{0}: T=T_{0}} \cdot \Delta T
$$

The dependence $\varepsilon_{\text {residual }}(\varphi)$ is determined by the equations similar to (39) and the dependence of $\varepsilon_{\text {residual }}(T)$ is determined by the equations similar to (20), (21), (27), (28), (30), (31), which includes the parameter $\tau$ (period/duration of temperature relaxation of the $\mathrm{CCPB}$ ), being the function $T(\mathrm{~s})$. Consequently, derivatives $\partial \varepsilon_{r} /\left.\partial \varphi\right|_{\varphi=\varphi_{0}: T=T_{0}}$ and $\partial \varepsilon_{r} /\left.\partial T\right|_{\varphi=\varphi_{0}: T=T_{0}}$ can be easily determined (here $\varepsilon_{r} \quad(\varphi, T)$ is introduced as total residual deformation due to changes of $T$ and $\varphi$ in the CCPB's environment).

During the use of mechatronic systems for controlling the artificial microclimate of museum rooms, appropriate digital signals (from the $\varphi$ and $T$ room sensors via ADC) are received by controllers performing operation, control and regulation functions of this microclimate. These signals adjust the PC program (or control unit of air conditioners) and provide (via ADC and actuator module) the high-quality functioning of working elements of the artificial microclimate's system in rooms (open/close dampers, nozzles, humidifiers, etc.). The quality of such functioning is determined by the following criterion:

$$
\left\{\varepsilon_{\text {residual }}^{\left(U^{*}\right)}(\varphi, T)-\varepsilon_{\text {residual }}^{(0)}\left(\varphi_{0}, T_{0}\right)\right\}^{2} \rightarrow 0,
$$


or:

$$
\left\{\left.\frac{\partial \varepsilon_{r}}{\partial \varphi}\right|_{\varphi=\varphi_{0} ; T=T_{0}} \cdot \Delta \varphi+\left.\frac{\partial \varepsilon_{r}}{\partial T}\right|_{\varphi=\varphi_{0} ; T=T_{0}} \cdot \Delta T\right\}^{2} \rightarrow 0
$$

The values of the function $\varepsilon_{r}(\varphi, T)$ can be found due to equations describing the dependence $\varepsilon_{r}(\varphi)$ and $\varepsilon_{r}(T)$. The departures of temperature $\Delta T$ and humidity $\Delta \varphi$ from $T_{0}$ and $\varphi_{0}$ (stipulated by state regulations for the temperature-humidity conditions of museum rooms, air velocity in such rooms, etc.) are tracked by special sensors (detectors, sensing elements) located in these museum rooms and are transferred in real time via ADC to the computer control system of rooms' artificial microclimate to adjust this device's program.

\section{Conclusions}

1. The residual deformations in the CCPB are generated by elastic deformations and follow them with respect to their rate of increase and residual deformations' value will depend on the duration of effect and value of elastic deformations, changes of temperature and humidity in the material interacting with the environment.

2. Within the framework of Zener model, the dependence of the CCPB's residual deformations on the temperature of the environment was determined analytically.

3. The use of spline approximations (splines of the third order with respect to $\varphi$ ), allows to determine with a high degree of accuracy the dependence of the CCPB's residual deformations on the relative humidity of the environment (air).

4. The criterion for quality control of the artificial microclimate system used for museum rooms, which can be realized using mechatronic control elements of the above system's components, is proposed.

5. The results obtained in the frame of this research can be further used to precise and improve the existing methods for calculating the parameters of mechatronic control systems operating the artificial climate of museum rooms both at such systems' designing stage and in the process of their real exploitation. 


\section{Bibliography}

[1.] O.Y. Ishlinskiy, "Applied Mathematics and Mechanics.” vol. 4, iss. 1, 1940.

[2.] O.R. Rzhanitsin, Nekotorye voprosy mekhaniki sistem, deformiruyushchikhsya vo vremeni [Some Issues of Systems' Mechanics Deforming in Time], Gostekhoretizdat, Moskow, 1949 (in Russian).

[3.] E.S. Sorokin, "K teorii vnutrennego treniya pri kolebaniyakh uprugikh sistem," [To the Theory of Internal Friction during Elastic Systems Vibrations] TsNIISK, Moskow, 1960, p. 132 (in Russian).

[4.] Uprugost' i neuprugost' metallov. Sbornik perevodov. [Elasticity and Non-elasticity of Metals. Translations Collection], Inostrannoi literatury Publ., Moskow, 1960 (in Russian).

[5.] Zener C. "The Physical Review," V.52, No. 3, 1937, p. 230.

[6.] L.D. Landau and E.M. Lifshitz, Mekhanika sploshnykh sred [Continuum Mechanics], Gostekhizdat, Moskow, 1953.

[7.] A.V. Lykov, Teoriya teploprovodnosti [Theory of Heat Conduction], Vysshaya shkola, Moskow, 1967, p. 599 (in Russian).

[8.] I.M. Ozarkiv et al., Kontrol' napruzheno-deformovanogo stanu i vologosti derevyny v teplomasoobminnikh protsesakh sushinnya [Control of Tense-Deformed State and Moisture of Wood in Heat and Mass Transfer during Drying Process], Scientific Bulletin of UNFU, vol. 28, no 10, 2018, pp. 81-84 (in Ukrainian).

[9.] A.V. Lykov, Teoriya sushki [Theory of drying], Energoizdat, Moskow-Leningrad, 1963, p. 417 (in Russian).

[10.] A.V. Kiselev and V.M. Dreving, ed., Eksperimental'nye metody $v$ adsorbtsyi $i$ molekulyarnoy khromatografii [Experimental Methods in Adsorption and Molecular Chromatography], MGU Publ., Moskow, 1973, p. 447 (in Russian).

[11.] S. J. Gregg and K. S. W. Sing, Adsorbtsiya, udel'naya poverkhnost', poristost' [Adsorption, Surface Area and Porosity], (Transl. from English), $2^{\text {nd }}$ ed., Mir Publ., Moskow, 1984, p. 306 (in Russian).

[12.] S. Brunauer, Adsorbtsiya gazov i parov [The Adsorption of Gases and Vapours], (Transl. from English), Inostrannoi literatury Publ., Moskow, 1948, p. 352 (in Russian).

[13.] Lutsyk R.V. et al., Teplomassoobmen pri obrabotke tekstil'nykh materialov [Heat and Mass Transfer during Textiles Processing], Naukova dymka, Kyiv, 1993, p. 344 (in Russian). 